\title{
Activation of AtMPK9 through autophosphorylation that makes it independent of the canonical MAPK cascades
}

\author{
Affiliations \\ Szilvia K. Nagy*, Zsuzsanna Darula†, Brigitta M. Kállai*, László Bögre ‡, Gábor Bánhegyi*, Katalin \\ F. Medzihradszky†, Gábor V. Horváth §, Tamás Mészáros*, II \\ *Department of Medical Chemistry, Molecular Biology and Pathobiochemistry, Semmelweis \\ University, H-1444 Budapest, POB 260, Hungary \\ †Proteomics Research Group, Biological Research Center of the Hungarian Academy of Sciences, \\ Szeged, H-6701, Szeged, POB 521, Hungary \\ $\ddagger$ School of Biological Sciences, Centre for Systems and Synthetic Biology, Royal Holloway, \\ University of London, Egham, Surrey, TW20 0EX, UK \\ §Institute of Plant Biology, Biological Research Centre of the Hungarian Academy of Sciences, H- \\ 6701, Szeged, POB 521, Hungary \\ II Technical Analytical Research Group of HAS, Szent Gellért tér 4, H-1111 Budapest, Hungary \\ Corresponding author: tamas.meszaros@med.semmelweis-univ.hu
}

\begin{abstract}
Mitogen activated protein kinases (MAPKs) are part of conserved signal transduction modules in eukaryotes that are typically organised into three-tiered kinase cascades. The activation of MAPKs in these pathways is fully dependent on the bisphosphorylation of the TXY motif in the T-loop by the pertinent dual-specificity MAPK kinases (MAPKKs). The plant AtMPK9 is a member of an atypical class of MAPKs. Representatives of this MAPK family have TDY phosphoacceptor site, a long C-terminal extension, and lack the common MAPKK binding docking motif. Here, we present multiple in vitro and in vivo data that AtMPK9 is activated independently of any upstream MAPKKs but it is activated through autophosphorylation. We mapped the autophosphorylation sites by mass spectrometry to the TDY motif and to the C-terminal regulatory extension. We mutated the phosphoacceptor sites on the TDY, which confirmed the requirement for bisphorylation at this site for full kinase activity. Next, we demonstrated that the kinase inactive mutant form of AtMPK9 is not transphosphorylated on the TDY site when mixed with an active AtMPK9, implying that the mechanism of the autocatalytic phosphorylation is intramolecular. Furthermore, we show that in vivo AtMPK9 is activated by salt and is regulated by okadaic acid-sensitive phosphatases. We conclude that the plant AtMPK9 shows similarities to the mammalian atypical MAPKs, ERK7/8 in terms of MAPKK-independent activation mechanism.
\end{abstract}

Key words: plant MAPK, TDY motif, atypical MAPK, MAPKK-independent, autophosphorylation, in vitro translation, AtMPK9

Abbreviations used: CD, common docking domain; GOF, gain-of-function; LC/MS/MS, liquid chromatography tandem mass spectrometry; LOF, loss-of-function; MAPK and MPK, mitogen activated protein kinase; MAPKK and MKK, MAPK kinase; MBP, myelin basic protein; p-ERK, phospho-extracellular regulated kinase;

Short (page heading) title: Autoactivation of a plant atypical MAPK, AtMPK9

Summary statement: AtMPK9, a plant-specific mitogen-activated protein kinase is autoactivated independently of canonical MAPK cascades. Phosphoacceptor amino acid residues of the activation loop are bisphosphorylated by intramolecular autophosphorylation. 


\section{Introduction}

Reversible protein phosphorylation at serine, threonine and tyrosine residues is a ubiquitous mechanism of signal transduction in all eukaryotes. The mitogen-activated protein kinase (MAPK or MPK according to the plant nomenclature) family of protein kinases provide prevalent mechanisms for transducing extracellular mitogen and stress stimuli into cellular responses. In accordance with their versatile physiological functions, the hundreds of identified MAPK substrates range from transcription factors to cytoskeletal proteins and they are involved in many aspects of cellular functioning [1].

MAPKs are serine/threonine protein kinases and phosporylate their targets within a minimal S/T-P motif upon activation. The evolutionary conserved core signalling module of MAPK pathways is composed of a three-tiered cascade of kinases that activate the subsequent kinase by phosphorylation. More specifically, the extracellular signal-sensing receptor activates the most upstream component of the module, typically the serine/threonine MAPK kinase kinase (MAP3K or MEKK) that in turn phosphorylates MAPK kinase (MAP2K, MKK, or MEK). The activated dual-specificity MKK effectively phosphorylates both the tyrosine and threonine residues at the conserved TXY motif in the activation loop of the MAPK. The active MAPK than phosphorylates the pertinent protein substrates which leads to cellular responses.

The majority of the MAPKs are activated within such canonical MAPK cascades and therefore classified as conventional MAPKs. The mammalian representatives of these MAPKs are extracellular signal-regulated kinases (ERK1/ERK2), c-Jun N-terminal kinases (JNKs), p38 and ERK5, which are phosphorylated by MKK1/MKK2, MKK4/MKK7, MKK3/MKK6 and MKK5, respectively [2]. The conventional MAPKs are all characterized by the presence of the TXY motif in their activation loop of the catalytic domain and require phosphorylation of both phosphoacceptor amino acids to become fully active.

Another group of MAPKs, referred to as atypical MAPKs, are activated through a distinct, MKK-independent activation mechanism. In animal cells ERK3/4 and ERK7/8 belong to the atypical MAPKs. These ERKs show low level of homology, but similar structural organization, possessing a unique C-terminal extension with largely unknown functions [3]. Besides the C-terminal extension, ERK3/4 differs from classical MAPKs in phosphorylation requirements of their activation loop too. Both kinases lack the tyrosine phosphoacceptor site of the conserved TXY motif, but contain a SEG amino acid triplet and phosphorylation of the single serine residue within this motif is sufficient for their activation [4]. ERK3/4 cannot be phosphorylated and activated by any of the MKKs but instead they are phosphorylated by a group of I p21-activated kinases (I PAKs) which act as upstream activators for these MAPKs [5]. ERK7/8 resemble to ERK1/2 and ERK5 in having a TEY phosphoacceptor motif in the activation loop [6,7]. Despite to the canonical TXY motif, nor the rat ERK7 neither its human homologue, ERK8 require an upstream MKK for activation, but both residues are autophosphorylated involving an autocatalytic mechanism [8,9].

Plants feature the most extended MAPK family of all eukaryotes implying the versatility of these signalling mechanisms to regulate cellular functions. The plant MPKs were classified into four phylogenetic groups, designated A-D [10]. Comparing the sequences at the activation loops, MPKs fall into two types: those carrying the amino acid motif TEY (A-C group) and those with a plant-specific TDY phosphorylation motif (D group). The TEY-type MPKs possess the evolutionary conserved common docking (CD) domain, a characteristic feature of classical MAPKs. The CD contains an amino acid sequence with two adjacent acidic residues that are crucial for interaction with substrates and regulators of MAPKs such 
as MKKs and phosphatases [11]. In accordance, it was shown that MPKs of the A-C groups can interact with and activated by pertinent MKKs and function in canonical MAPK pathways [12]. The D group of plant MAPKs that have TDY type of activation loop also differ from the classical MAPKs in several other aspects. Similarly to atypical MAPKs, they have a long Cterminal extension and a diverged CD domain, wherein certain acidic residues are replaced with basic amino acids [13]. A recent study suggested that a member of the D group MPK, AtMPK8 is regulated both by MKK3 and by calmodulin [14]. However, it was not shown whether the activation is down to direct phosphorylation of the threonine and tyrosine residues at the TDY motif by MKK3.

Here, we show that the D-type Arabidopsis MAPK, AtMPK9 is phosphorylated both at threonine and tyrosine residues of the TDY motif, which is required for the activity of the kinase. This phosphorylation relies on an intramolecular autocatalytic mechanism rather than on an upstream kinase. We identified serine residues of the C-terminal extension that also gets autophosphorylated. We also demonstrated that AtMPK9 is activated by salt treatment and regulated by okadaic acid-sensitive phosphatases in vivo.

\section{Materials and methods}

Plasmid construction, in vitro mutagenesis

The AtMPK9 (AT3G18040) gene was amplified from cDNA library using iProof HighFidelity DNA polymerase (BioRad). The PCR products were inserted into the pEU3-NIIHLICNot vector by ligation independent cloning (LIC) as described in [15]. This vector is a modification of what was described by Bardóczy et al. for wheat germ translation with $\mathrm{His}_{6}$ affinity tag [16]. The myc-tagged, constitutively active, gain of function (GOF) mutant form of AtMKK4 was inserted into pEU3-NII-Gateway destination vector by Gateway cloning. PCR fragments of AtMPK9 mutants were cloned into pRT-HA vector [17] using NotI and NcoI restriction endonucleases (Fermentas) for protoplast transformation. The myc-tagged, GOF-MKK constructs were recloned into pK2GW7 vector [18] by Gateway conversion. Site directed mutagenesis of AtMPK9 were performed by QuikChange ${ }^{\circledR}$ Site-Directed Mutagenesis Kit (Stratagene) following the provided manual. XL-10 GOLD Escherichia coli competent cells (Stratagene) were transformed and plasmids were purified with PureLink Quick Plasmid Miniprep Kit (Life Technologies). All constructs and site directed mutagenesis were confirmed by sequencing. Plasmids for transient protoplast expression studies were purified by HiSpeed Plasmid Maxi Kit (Qiagen).

\section{In vitro transcription and cell-free translation}

To increase the efficiency of the in vitro transcription reaction, we generated linearized template DNA constructs with NotI (Fermentas). Transcription was performed by TranscriptAid $^{\mathrm{TM}}$ T7 High Yield Transcription Kit (Fermentas) according to the suggested protocol. The mRNA was precipitated by ammonium acetate/ethanol mixture, dissolved in $1 \mathrm{x}$ SUB-AMIX and stored at $-80^{\circ} \mathrm{C}$. Quality and quantity of mRNAs was verified on agarose gel electrophoresis. Cell-free translation was carried out in $20.6 \mu 1$ final volume by addition of 5 $\mu \mathrm{L}(15 \mu \mathrm{g}) \mathrm{mRNA}, 10 \mu \mathrm{L} \mathrm{WEPRO}^{\circledR}$ (Cell Free Sciences) solution, $0.8 \mu \mathrm{L}$ creatine kinase $(1$ $\mathrm{mg} / \mathrm{mL}$ ) and $5 \mu \mathrm{L} 1 \mathrm{x}$ SUB-AMIX. Additional $0.5 \mu \mathrm{l}$ of myc-GOF-AtMKK4 mRNA was added to the translation mixture for co-translation of myc-GOF-AtMKK4 with His 6 -AtMPK6 leading to the active $\mathrm{His}_{6}$-AtMPK6. The translation solution was underlayed to $206 \mu \mathrm{L} \mathrm{SUB}-$ AMIX in a sterile 96-well plate and the reaction was incubated for 20 hours at $20^{\circ} \mathrm{C}$ [15].

Plant cell culture, protoplast transformation and treatments 
Columbia (Col-0) Arabidopsis thaliana root cell suspension culture was maintained according to Mathur and Koncz [19]. Isolation of protoplast and polyethylene glycol-mediated protoplast transformation were carried out as described by Anthony et al [20]. We used $5 \mu \mathrm{g}$ plasmid DNAs to transform $2 \times 10^{5}$ protoplasts, and then cells were cultured for $16 \mathrm{~h}$ in dark at room temperature before harvesting. In case of myc-GOF-AtMKK co-transformation, 5:1 ratio was applied; $5 \mu \mathrm{g}$ of AtMPK9-HA coding vector and $1 \mu \mathrm{g}$ of different myc-GOF-MKK constructs. For stress activation of AtMPK9 $500 \mathrm{nM}$ flg22, $250 \mathrm{mM} \mathrm{NaCl}, 20 \mathrm{nM} \mathrm{H}_{2} \mathrm{O}_{2}$ and cold treatment were implemented for 10 and 30 minutes. Phosphatase inhibitor treatment was made by addition of $100 \mathrm{nM}$ or $1 \mu \mathrm{M}$ okadaic acid (Sigma-Aldrich) and $100 \mu \mathrm{M}$ or $1 \mathrm{mM}$ sodium orthovanadate.

Protein purification, phosphatase treatment, TEV protease cleavage

In vitro translated proteins were purified by affinity chromatography on $10 \mu 1$ TALON $^{\circledR}$ Magnetic Beads (Clontech). $50 \mathrm{mM}$ sodium phosphate buffer complemented with $10 \mathrm{mM}$ imidazole, $300 \mathrm{mM} \mathrm{NaCl}, 0.1 \%$ Triton- $X$ was used as wash and binding buffer. The resin was washed three times after $1 \mathrm{~h}$ incubation with total translation mixture at room temperature. Phosphatase treatment was carried out with $\lambda$ Protein Phosphatase (New England Biolabs) for 30 minutes at $30^{\circ} \mathrm{C}$, and then washed three times with PBS, $\mathrm{PH}$ 7.4. TEV protease was affinity purified after bacterial protein expression using pTH24_TEV construct [21]. TEV protease cleavage was performed overnight and at $4{ }^{\circ} \mathrm{C}$ in $50 \mu$ final volume with addition of $2.5 \mu \mathrm{l} 20 \mathrm{X}$ TEV Buffer (1 M Tris-HCl, $\mathrm{pH}$ 8.0, 10 mM EDTA), $0.5 \mu 10.1 \mathrm{M}$ DTT, $10 \mu 1$ TEV protease to affinity purified proteins. Protein concentration was measured by Bradford reagent. Protoplast expressed AtMPK9-HA was purified with anti-HA agarose beads (SigmaAldrich) after sonication of protoplasts in $100 \mu \mathrm{l}$ Lacus buffer as described in [22].

\section{In vitro protein kinase assays}

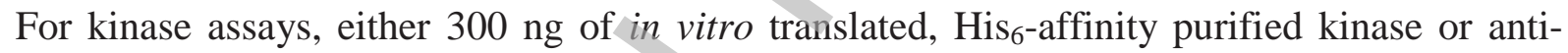
HA-purified, in vivo expressed kinase from $30 \mu \mathrm{g}$ total protein of protoplasts were used. The assay was carried out in $20 \mathrm{mM}$ HEPES, pH 7.5, $100 \mu \mathrm{M}$ ATP, $1 \mathrm{mM}$ DTT, $15 \mathrm{mM} \mathrm{MgCl}_{2}, 5$ mM EGTA, $5 \mu \mathrm{Ci}\left[\gamma_{-}{ }^{32} \mathrm{P}\right] \mathrm{ATP}$ and $1 \mathrm{mg} / \mathrm{ml}$ myelin basic protein (Sigma-Aldrich) as a MAPK substrate in $16 \mu \mathrm{l}$ final volume for 30 minutes at room temperature, then stopped by addition of 5x Laemmli SDS loading buffer. Samples were loaded onto a 15\% SDS-PAG. The gel was fixed, stained with Coomassie Blue, dried and analyzed by autoradiography.

\section{Immunoblotting}

Proteins were separated using 10\% SDS-PAGE, and subsequently blotted to PVDF membrane (Millipore) and blocked in 5\% milk, PBS, 0.2\% TWEEN-20, pH 7.4 solution for 1 hour. In the case of immunoblotting with p-ERK antibody (Cell Signaling), proteins were transferred onto nitrocellulose membrane and blocking was performed in phosphate free solution: $5 \%$ BSA, TBS, 0.2 \% TWEEN-20, pH 7.4. Anti-HA-peroxidase (Roche), anti-c-myc-peroxidase (Roche), goat anti-rabbit IgG-HRP (Santa Cruz Biotechnology) and p-ERK (Cell Signaling) antibodies were used. When multiple antibodies were used in successions, the membrane was stripped with $20 \mathrm{ml}$ freshly made stripping buffer (200 mM glycine, 1\% TWEEN-20 and $0.1 \%$ SDS,pH 2.2).

\section{LC-MS/MS analysis}

Phosphorylation reaction mixtures were separated by 10\% SDS-PAGE, bands corresponding to AtMPK9 protein were excised from the gel and subjected to in-gel digestion using sidechain-protected porcine trypsin [23]. Approximately 20\% of the resulting peptide mixtures 
were subjected directly to LC-MS/MS analyses and the other $80 \%$ was enriched for phosphopeptides using $\mathrm{TiO}_{2}$ [24]. LC-MS/MS experiments were performed in "triple play" acquisition mode using a Thermo LCQ-Fleet ion trap mass spectrometer on-line coupled to an Eldex nanoHPLC system. Raw data were converted into peak lists using the Mascot Distiller software applying default LCQ_Fleet opt processing parameters. Database searches were performed using the ProteinProspector search engine (version 5.12.2) with the following parameters: database: Swissprot or user database containing the sequences of wild-type and mutant MPK9s'; enzyme: trypsin allowing 1 missed cleavage; mass accuracies: 0.6 Da for precursors and $1 \mathrm{Da}$ for fragment ions (both monoisotopic); modifications: fixed: carbamidomethylation of Cys's, variable: methionine oxidation, pyroglutamic acid formation from peptide N-terminal Gln residues and acetylation of protein N-termini allowing maximum 2 modifications per peptide. For phosphopeptide identifications the same parameters were applied except that 2 missed cleavages and phosphorylation of Ser/Thr/Tyr residues with maximum 3 variable modifications per peptide were allowed. Acceptance parameters: score: 22 and 15, E value: 0.01 and 0.05 for protein and peptide identifications, respectively, SLIP (site localization in the peptide) score: 6 [25]. Samples were also analyzed in a data-dependent acquisition mode using a Waters Q-TOF Premier mass spectrometer online coupled to a nanoAquity UPLC system. From the raw data peaklists were generated by the Mascot Distiller software using default QTOF.opt processing parameters. Database searches were conducted as described above except that mass accuracies were set to 20 and $100 \mathrm{ppm}$ for precursor and fragment ions (both monoisotopic), respectively allowing 45 ppm systematic error for precursor ions; and ESI-Q-TOF was specified as instrument. All phosphopeptide identifications were inspected manually.

\section{Results}

The in vitro translated AtMPK9 has an intrinsically high kinase activity

The His ${ }_{6}$-tagged AtMPK6 and AtMPK9 were taken as representatives of A and D groups of plant MPKs and were synthesized by wheat-germ based in vitro translation. The purified AtMPK6 and AtMPK9 showed a clear difference in the migration pattern on SDS-PAG. In contrast to the single, definitive band of AtMPK6, gel electrophoretic separation of AtMPK9 produced multiple protein bands that shifted upwards. Incubation of AtMPK9 with $\lambda$ phosphatase converted this smeared migration pattern into a single band on SDS-PAG (Figure $1 \mathrm{~A})$, suggesting that the differently migrating forms are due to phosphorylation. In addition, AtMPK9 had a significantly higher myelin basic protein (MBP) kinase activity than the in vitro translated AtMPK6 (Figure 1B).

Bisphosphorylation of the TXY phosphoacceptor motif in the T-loop is known to be fully required for MAPK activation [22]. To determine the phosphorylation state of the TXY motif on AtMPK6 and AtMPK9, we used a polyclonal antibody that was developed against the double phosphorylated TEY amino acid triplet of the animal ERK1. The in vitro translated AtMPK6 was only recognized by phospo-ERK (p-ERK) antibody when the cognate constitutively active gain of function (GOF) GOF-AtMKK4 was co-translated with it, suggesting that AtMKK4 is inevitable for the phosphorylation of TEY on AtMPK6. On the contrary, the in vitro translated recombinant AtMPK9 was recognized by the same antibody without the need to add MKKs (Figure 1B). We confirmed the phosphorylation of the threonine and tyrosine residues within the characteristic TDY motif of AtMPK9 by mass spectrometry (MS) analysis. The corresponding [173-191] peptide to TDY was present both in mono- and bisphosphorylated forms and with MS/MS analysis we could assign the phosphorylation to Tyr-187 in the monophosphorylated peptide (Suppl. figure 1). 


\section{The phosphorylation of TDY motif on AtMPK9 is fully required for the kinase activity}

The majority of MAPKs require double phosphorylation of the TXY motif to be fully active. We exchanged in combinations the threonine and tyrosine phosphoacceptor sites of the TDY motif of AtMPK9 to non-phosporylatable alanine and phenylalanine by in vitro mutagenesis to address whether the activity of AtMPK9 depends on bisphosphorylation of the TDY phosphoacceptor site. Single T185A, Y187F and double T185A/Y187F mutants of AtMPK9 were produced by in vitro translation. All three of these mutant AtMPK9 forms migrated as distinct bands on SDS-PAG, whereas the wild-type AtMPK9 was separated in a diffuse band as was observed before (Figure 2A). To confirm the lack of phosphorylation at the TXY motif of these three mutant AtMPK9 forms, tryptic digests of the proteins were analyzed by datadependent LC/MS/MS. The MS data verified the amino acid exchanges performed by in vitro mutagenesis. More importantly, phosphotyrosine and phosphothreonine were detected in the T185A and Y187F mutants, respectively (Figure 2B) suggesting that the single replacement of either threonine or tyrosine in the TDY motif did not influence the phosphorylation of the non-exchanged phosphoacceptor site. Using these mutant AtMPK 9 forms, we addressed how the phosphorylation state of the TDY motif impacts on the kinase activity of AtMPK9. None of the mutant AtMPK9 forms had any detectable activity towards MBP in vitro (Figure 2A, bottom panel). These results suggested that phosphorylation of both phosphoacceptor amino acids in the TDY motif is essential for the activation of AtMPK9.

\section{Activation of AtMPK9 does not depend on MKKs}

In plants, there is no evidence for activation of MPKs that does not involve upstream MKKs. MAPK activation by MKKs typically involves their specific interactions. However, in agreement with published systematic yeast two hybrid interaction screens among MKKs and MPKs, we also could not detect interaction of AtMPK9 with MKKs by yeast two hybrid assay (data not shown and [26]). Alternatively, AtMPK9 interaction with and activation by MKKs might rely on yet unidentified scaffold proteins present in Arabidopsis cells [27]. To examine this possibility, we expressed HA-tagged AtMPK9 and constitutively active mutants of Arabidopsis MKKs in transformed protoplast. First, we set out to determine the physiologically relevant stimuli that activate AtMPK9 in vivo. To do this, we treated cells with salt, cold, $\mathrm{H}_{2} \mathrm{O}_{2}$, and the pathogenic elicitor, flg22, immunoprecipitated the tagged AtMPK9-HA protein and performed in vitro kinase activity measurements. All tested stress conditions, except cold increased the AtMPK9 kinase activity in 10 minutes and salt treatment showed to be the most pronounced effector (data not shown). Accordingly, we used $10 \mathrm{~min}$ treatment with $250 \mathrm{mM}$ salt to elicit kinase activity of AtMPK9 in the subsequent experiment. Next, we co-transformed AtMPK9 with a panel of constitutively active, gain-of-function MKKs (GOF-MKK), representing each plant MKK subgroup. The kinase activity-elevation of AtMPK9 was negligible with any of the GOF-MKKs in comparison to the salt treatment of protoplasts (Figure 3). The bisphosphorylated TDY motif and the high catalytic activity of in vitro translated AtMPK9, the lack of interaction with MKKs and the inability to be activated by GOF-MKKs in cells all indicate an MKK independent but autophosphorylation-dependent activation mechanism.

\section{AtMPK9 is activated by autophosphorylation}

In order to see whether the phosphorylation of the T-loop is indeed dependent on the kinase activity of AtMPK9, we produced a catalytically inactive, loss-of-function (LOF) mutant form of AtMPK9 by replacing one of the lysines of the ATP binding site. SDS-PAGE separation of the affinity-purified LOF-AtMPK9 showed a single distinct band, a migration pattern which was similar to that of the phosphoacceptor-site mutants (Figure 4A). We confirmed by in vitro 
kinase assay that the LOF-AtMPK9 is inactive and next we looked to see whether the phosphorylation of the TDY motif is dependent on the AtMPK9 kinase activity. The active in vitro translated AtMPK9 but not the LOF-AtMPK9 was detected by the p-ERK antibody. The lack of TDY phosphorylation of the LOF-AtMPK9 on one hand rules out that there is an unknown protein kinase in the wheat germ extract used for in vitro translation that phosphorylates AtMPK9, while on the other hand suggests that AtMPK9 activity is required for TDY phosphorylation, likely through an autocatalytic reaction (Figure 4A). MS analysis was in agreement with the data obtained by immunoblot with p-ERK antibody; no phosphorylated tryptic peptide spanning the TDY motif [173-191] of LOF-AtMPK9 could be detected.

To corroborate the conclusion that the in vitro translated AtMPK9 is autophosphorylated, we set out to determine whether or not the LOF-AtMPK9 was phosphorylated on the TDY sites in vivo. Protoplasts were transformed to express WT-AtMPK9 and LOF-AtMPK9 and treated with salt. Western blot analysis with p-ERK antibody demonstrated that the TDY motif of WT-AtMPK9 became phosphorylated under stress conditions (Figure 4B). In contrast, the treated and non-treated LOF samples were indistinguishable; none of them were detectable with the p-ERK antibody. In vitro kinase activities of HA-immunoprecipitated samples were also measured and the obtained data were in accordance with the Western blot results; only the salt-treated WT-AtMPK9 was active to phosphorylate MBP.

It is known that MAPKs are negatively regulated by multiple phosphatases of Tyr-, Ser/Thr-, and dual-specificities. To reveal whether or not phosphatases regulate the TDY phosphorylation and activity of AtMPK9 in vivo, we treated protoplasts expressing WTAtMPK9 or LOF-AtMPK9 with two different phosphatase inhibitor, okadaic acid inhibiting PP2A, PP2B and to a lesser extent PP1 and with orthovanadate that inhibits Tyr and dualspecificity phosphatases. Analysing TDY phosphorylation state with p-ERK antibody showed that okadaic acid treatment led to a dramatic increase in TDY phosphorylation of WTAtMPK9 (Figure 5), while orthovanadate had hardly any effect (data not sown). The activity of WT-AtMPK9 in these samples paralleled the TDY phosphorylation. Importantly, none of the phosphatase inhibitor treatments led to TDY loop phosphorylation of the kinase inactive, LOF-AtMPK9 mutant. Collectively, these results show that AtMPK9 is activated by autophosphorylation and this is regulated directly or indirectly by okadaic acid sensitive phosphatases in vivo.

\section{AtMPK9 is phosphorylated by an intramolecular mechanism}

In most of the cases, autophosphorylation is an intermolecular reaction in which one kinase molecule phosphorylates another closeby kinase molecule, but intramolecular kinase autophosphorylation has also been described [28]. In order to distinguish between these two autocatalytic processes, first, we dephosphorylated the affinity-bead coupled, AtMPK9 by $\lambda$ phosphatase and tested whether it can still be autophosphorylated. In accordance with our previous results, phosphatase treatment resulted in a down-shift of WT-AtMPK9 on SDSPAG and migrated to the same position as the LOF-AtMPK9 (Figure 6A). Next, we run kinase reactions with the purified $\lambda$ phosphatase treated and untreated AtMPK9 samples and detected the phosphorylated forms on an autoradiogam. Both samples showed phopshorylation of AtMPK9 that was even stronger when WT-AtMPK9 was treated with the $\lambda$ phosphatase (Figure 6B upper panel). The MBP kinase activity again mirrored the level of autophosphorylation, indicating that the phosphorylation and activity of purified AtMPK9 was efficiently restored by an autocatalytic mechanism (Figure 6B bottom panel). Lastly, we set out to test whether an active AtMPK9 can transphosphorylate the kinase inactive LOFAtMPK9 mutant. We immobilized LOF-AtMPK9 kinase to beads, added active AtMPK9 to it 
and make the kinase reaction. Subsequently, we recovered the bead-immobilized LOFAtMPK9 and run on SDS-PAG. No phosphorylation could be detected on the autoradiogram, implying that the LOF-AtMPK9 mutant cannot be transphosphorylated by the active AtMPK9 (Figure 6B upper panel). These in vitro kinase experiments show that AtMPK9 is activated by intramolecular autophosphorylation.

\section{The C-terminal domain of AtMPK9 is also autophosphorylated at multiple sites}

The diffuse migration pattern of AtMPK9 hints that the kinase is phosphorylated on multiple sites. According to the LC/MS/MS data, the active and inactive forms of AtMPK9 hold different phosphorylation patterns in their C-terminal extensions. Beside the TDY motif, Ser401, Ser-443, and Ser-464 were also phosphorylated in the wild-type AtMPK9 but none of these residues were detected to be phosphorylated with the catalytically inactive LOFAtMPK9 mutant form. Interestingly, Ser-464 is followed by an alanine residue instead of a proline, implying that AtMPK9 is capable of phosphorylating serine residues that do not fulfil the S/T-P definition of MAPK phosphorylation sites (Suppl. figure 2).

\section{Discussion}

The hallmark of the conventional MAPKs is the TXY amino acid triplet at the activation loop that is phosphorylated on both phosphoacceptor residues by the cognate MAPKKs to fully activate the kinase. This double phosphorylation is accomplished by dual-specificity upstream MAPKKs in two steps, first the tyrosine then the threonine is modified that results in a fourmagnitude increase in the activity [29]. Selective activation of MAPKs is ensured by interaction between the C-terminally located CD of MAPKs and its complementary docking domain of the pertinent interacting proteins [11].

Several experiments have demonstrated that phosphorylation by MAPKKs is not the sole mechanisms of MAPK activation. Some of the atypical MAPKs have to be phosphorylated on a single serine residue by IPAKs for elicitation [5]. Other representatives of atypical MAPKs, such as ERK7/8 gain their activity by intramolecular autophosphorylation on both phosphoacceptor sites of the TXY motif [8,9]. Surprisingly, p38 $\alpha$, a conventional MAPK beside the prototypical MAPKK phosphorylation, also could be activated by an autocatalytic manner. This alternative activation mechanism is induced by direct interaction of $\mathrm{p} 38 \alpha$ with TGF-Beta Activated Kinase 1/MAP3K7 Binding Protein 1 (TAB1) that results in MAPKKindependent dual phosphorylation of the TGY motif of the activation loop [30]. These results hint that regulation of MAPKs is more sophisticated than it was previously anticipated, and authophosphorylation might also be a more general activation mechanism of MAPKs that can coexist with the canonical activation through MAPKKs.

The Arabidopsis genome encodes 20 MPKs but only a handful of them were studied in details. These include AtMPK3, AtMPK4 and AtMPK6 that belong to the classical MAPKs carrying the TEY motif in their activation loops. These A and B group members of MPKs were shown to be activated by dual specificity MKKs [31]. MPKs that belong the C group was also proved to be activated through the canonical activation mechanism involving an upstream MKK, AtMKK3 [32]. In comparison to the TEY motif containing plant MPKs, much less data is available for the structurally most different MPKs with TDY activation loop in the D group [14,32]. AtMPK8 is the only D group MAPKs that was studied and was shown to be activated through phosphorylation by AtMKK3 [14]. Surprisingly, the other suggested activation process - direct interaction with calmodulin- does not rely on phosphorylation of 
threonine and tyrosine residues of the T-loop since the calmodulin induced activity elevation is similar in wild-type and the TDY mutant form of AtMPK8.

In this study, we have shown important insights into the regulation of AtMPK9, another representative of D group MPKs. In contrast to AtMPK6, the in vitro translated AtMPK9 showed high kinase activity without the addition of upstream MKKs. This high kinase activity was accompanied by dual phosphorylation of the TDY motif of the activation loop as it was demonstrated by immunoblot using p-ERK antibody. Phosphorylation of both phosphoacceptor amino acids within the TDY motif is essential for kinase activity since in vitro mutagenesis of either the threonine or tyrosine residue drastically reduced the activity of in vitro translated AtMPK9 mutants, even though non-mutated residues were phosphorylated according to MS/MS analysis. The MKK independent activation mechanism has been corroborated by in vivo studies using transformed Arabidopsis protoplasts. AtMPK9 was activated upon salt treatment when expressed in protoplasts, while none of the constitutively active mutant MKKs were able to activate AtMPK9. Furthermore, contrary to wild-type AtMPK9, the protoplast-expressed inactive mutant version of AtMPK9 was not recognized by p-ERK antibody following salt treatment, again indicating that the T-loop phosphorylation is an autocatalytic process. Application of phosphatase inhibitors provided an additional line of evidence for autophosphorylation of AtMPK9 and its regulation by phosphatases. Treatment of cells expressing wild-type AtMPK9 and LOF-AtMPK9 resulted in dramatically increased phosphorylation and activation of wild-type AtMPK9, while the phosphorylation of TDY motif in the kinase inactive LOF-AtMPK9 was insignificant following okadaic acid treatment. Finally, mass spectrometry analysis of in vitro translated and affinity-purified AtMPK9 complex failed to indentify any interacting, endogenous protein kinases of wheat germ extract (data not shown). These results collectively suggest that activation of AtMPK9 does not rely on upstream kinase, but involves an autophosphorylation mechanism and signalling inputs by protein phosphatases. According to our in vitro kinase activity assays, this autophosphorylation is intramolecular rather than intermolecular since the inactive LOFAtMPK9 mutant was not phosphorylated when mixed with an active AtMPK9. LC/MS/MS analysis of the tryptic digests demonstrated that the $\mathrm{C}$ - terminus of the kinase is also autophosphorylated: 3 serines at this unstructured domain were phosphorylated, but only with the wild-type AtMPK9. Interestingly, one of these residues does not fulfil the minimal requirement of the MAPK phosphorylation motif since the modified serine is not followed by a proline. These data imply that AtMPK9 has a broader phospho-site recognition specificity that what we know for canonical MAPKs.

A systematic screen for the interactions of MKKs and MPKs using yeast two hybrid failed to identify MKK partners for most D-type MPKs including AtMPK9 [26]. At the same time, our in vivo AtMPK9 activation experiments in transformed protoplasts suggested that AtMPK9 does not rely on any MKKs. According to our results, the in vitro translated AtMPK9 is activated by an intramolecular autocatalytic mechanism. This is in contrast to what has been suggested for AtMPK8, a close member within the D group, which is in parallel and independently activated by upstream MKK and calmodulin [14]. AtMPK9 activation is not dependent on MKKs and is strictly reliant on the autophosphorylation of both phosphoacceptor residues of the TDY amino acid triplet. The D-type MPKs display high homology in their kinase domains but are divergent in their C-termini (Suppl. figure 3). Therefore, representatives of this kinase group might be regulated by diverse mechanisms and the C-terminal domain might be a crucial component of controlling the activity of D-type MPKs. Considering this putative function of the C-terminus, phosphorylation of serines in this domain of AtMPK9 is also expected to have a physiological function in regulating the 
kinase activity, but further work is necessary to determine the biochemical mode of this regulation.

\section{Author contribution}

Experimental work and experimental design was carried out by Zsuzsanna Darula, Gábor V. Horváth, Brigitta M. Kállai, Tamás Mészáros, and Szilvia K. Nagy. Gábor Bánhegyi, László Bögre, Gábor V. Horváth, and Tamás Mészáros designed the study. Katalin F. Medzihradzky, Laszlo Bogre and Tamás Mészáros wrote the paper.

\section{Acknowledgments}

We thank Róbert Dóczi for providing myc-GOF-AtMKK vector constructs.

\section{Funding}

This work was supported by Hungarian Scientific Research Fund (K69187, IN76674, and NN111085)

\section{References}

1 Imajo, M., Tsuchiya, Y. and Nishida, E. (2006) Regulatory mechanisms and functions of MAP kinase signaling pathways. IUBMB Life 58, 312-317.

2 Cargnello, M. and Roux, P. P. (2011) Activation and function of the MAPKs and their substrates, the MAPK-activated protein kinases. Microbiol. Mol. Biol. Rev. 75, 50-83.

3 Coulombe, P. and Meloche, S. (2007) Atypical mitogen-activated protein kinases: structure, regulation and functions. Biochim. Biophys. Acta 1773, 1376-1387.

4 Déléris, P., Rousseau, J., Coulombe, P., Rodier, G., Tanguay, P.-L. and Meloche, S. (2008) Activation loop phosphorylation of the atypical MAP kinases ERK3 and ERK4 is required for binding, activation and cytoplasmic relocalization of MK5. J. Cell. Physiol. 217, 778-788.

5 Déléris, P., Trost, M., Topisirovic, I., Tanguay, P.-L., Borden, K. L. B., Thibault, P. and Meloche, S. (2011) Activation loop phosphorylation of ERK3/ERK4 by group I p21-activated kinases (PAKs) defines a novel PAK-ERK3/4-MAPK-activated protein kinase 5 signaling pathway. J. Biol. Chem. 286, 6470-6478.

6 Abe, M. K., Kuo, W., Hershenson, M. B. and Rosner, M. R. (1999) Extracellular Signal-Regulated Kinase 7 (ERK7), a Novel ERK with a C-Terminal Domain That Regulates Its Activity, Its Cellular Localization, and Cell Growth 19, 1301-1312.

7 Abe, M. K., Saelzler, M. P., Espinosa, R., Kahle, K. T., Hershenson, M. B., Le Beau, M. M. and Rosner, M. R. (2002) ERK8, a new member of the mitogen-activated protein kinase family. J. Biol. Chem. 277, 16733-16743.

8 Abe, M. K., Kahle, K. T., Saelzler, M. P., Orth, K., Dixon, J. E. and Rosner, M. R. (2001) ERK7 is an autoactivated member of the MAPK family. J. Biol. Chem. 276, 21272-21279. 
9 Klevernic, I. V, Stafford, M. J., Morrice, N., Peggie, M., Morton, S. and Cohen, P. (2006) Characterization of the reversible phosphorylation and activation of ERK8. Biochem. J. 394, 365-373.

10 Ichimura, K., Shinozaki, K., Tena, G. and Sheen, J. (2002) Mitogen-activated protein kinase cascades in plants: a new nomenclature. Trends Plant Sci. 7, 301-308.

11 Tanoue, T., Adachi, M., Moriguchi, T. and Nishida, E. (2000) A conserved docking motif in MAP kinases common to substrates, activators and regulators. Nat. Cell Biol. 2, 110-6.

12 Rodriguez, M. C. S., Petersen, M. and Mundy, J. (2010) Mitogen-activated protein kinase signaling in plants. Annu. Rev. Plant Biol. 61, 621-649.

13 Dóczi, R., Okrész, L., Romero, A. E., Paccanaro, A. and Bögre, L. (2012) Exploring the evolutionary path of plant MAPK networks. Trends Plant Sci. 17, 518-525.

14 Takahashi, F., Mizoguchi, T., Yoshida, R., Ichimura, K. and Shinozaki, K. (2011) Calmodulin-dependent activation of MAP kinase for ROS homeostasis in Arabidopsis. Mol. Cell, Elsevier Inc. 41, 649-660.

15 Szilvia K. Nagy and Tamás Mészáros. (2014) Cell-Free Protein Synthesis. In Methods in Molecular Biology (Alexandrov, K., and Johnston, W. A., eds.), pp 231-243, Humana Press, Totowa, NJ.

16 Bardóczy, V., Géczi, V., Sawasaki, T., Endo, Y. and Mészáros, T. (2008) A set of ligation-independent in vitro translation vectors for eukaryotic protein production. BMC Biotechnol. 8, 32.

17 Dóczi, R., Brader, G., Pettkó-Szandtner, A., Rajh, I., Djamei, A., Pitzschke, A., Teige, M. and Hirt, H. (2007) The Arabidopsis mitogen-activated protein kinase kinase MKK3 is upstream of group $C$ mitogen-activated protein kinases and participates in pathogen signaling. Plant Cell 19, 3266-3279.

18 Karimi, M., Inzé, D. and Depicker, A. (2002) GATEWAY vectors for Agrobacteriummediated plant transformation. Trends Plant Sci. 7, 193-195.

19 Mathur, J. and Koncz, C. (1998) Establishment and maintenance of cell suspension cultures. Arab. Protoc. 82, 27-30.

20 Anthony, R. G., Henriques, R., Helfer, A., Mészáros, T., Rios, G., Testerink, C., Munnik, T., Deák, M., Koncz, C. and Bögre, L. (2004) A protein kinase target of a PDK1 signalling pathway is involved in root hair growth in Arabidopsis. EMBO J. 23, $572-581$.

21 Li, Y. and Sousa, R. (2012) Expression and purification of E. coli BirA biotin ligase for in vitro biotinylation. Protein Expr. Purif. 82, 162-167.

22 Bögre, L., Calderini, O., Binarova, P., Mattauch, M., Till, S., Kiegerl, S., Jonak, C., Pollaschek, C., Barker, P., Huskisson, N. S., et al. (1999) A MAP kinase is activated 
late in plant mitosis and becomes localized to the plane of cell division. Plant Cell 11, 101-113.

23 Rosenfeld, J., Capdevielle, J., Guillemot, J. C. and Ferrara, P. (1992) In-gel digestion of proteins for internal sequence analysis after one- or two-dimensional gel electrophoresis. Anal. Biochem. 203, 173-179.

24 Larsen, M. R., Thingholm, T. E., Jensen, O. N., Roepstorff, P. and Jørgensen, T. J. D. (2005) Highly selective enrichment of phosphorylated peptides from peptide mixtures using titanium dioxide microcolumns. Mol. Cell. Proteomics 4, 873-886.

25 Baker, P. R., Trinidad, J. C. and Chalkley, R. J. (2011) Modification site localization scoring integrated into a search engine. Mol. Cell. Proteomics 10, M111.008078.

26 Lee, J. and Huh, K. (2008) Comprehensive analysis of protein-protein interactions between Arabidopsis MAPKs and MAPK kinases helps define potential MAPK signalling modules. Plant Signal. Behav. 3, 1037-1041.

27 Kolch, W. (2005) Coordinating ERK/MAPK signalling through scaffolds and inhibitors. Nat. Rev. Mol. Cell Biol. 6, 827-837.

28 Cole, A., Frame, S. and Cohen, P. (2004) Further evidence that the tyrosine phosphorylation of glycogen synthase kinase-3 (GSK3) in mammalian cells is an autophosphorylation event. Biochem. J. 377, 249-55.

29 Ferrell, J. E. and Bhatt, R. R. (1997) Mechanistic Studies of the Dual Phosphorylation of Mitogen-activated Protein Kinase. J. Biol. Chem. 272, 19008-19016.

30 Ge, B., Gram, H., Di Padova, F., Huang, B., New, L., Ulevitch, R. J., Luo, Y. and Han, J. (2002) MAPKK-independent activation of p38alpha mediated by TAB1-dependent autophosphorylation of p38alpha. Science 295, 1291-1294.

31 Colcombet, J. and Hirt, H. (2008) Arabidopsis MAPKs: a complex signalling network involved in multiple biological processes. Biochem. J. 413, 217-26.

32 Jammes, F., Song, C., Shin, D., Munemasa, S., Takeda, K., Gu, D., Cho, D., Lee, S., Giordo, R., Sritubtim, S., et al. (2009) MAP kinases MPK9 and MPK12 are preferentially expressed in guard cells and positively regulate ROS-mediated ABA signaling. Proc. Natl. Acad. Sci. U. S. A. 106, 20520-5.

33 Biemann, K. (1990) Nomenclature for Peptide Fragment Ions (Positive Ions)*. Methods Enzymol. 193, 886-887.

34 Zhang, Y. (2008) I-TASSER server for protein 3D structure prediction. BMC Bioinformatics 9, 40.

35 Pettersen, E. F., Goddard, T. D., Huang, C. C., Couch, G. S., Greenblatt, D. M., Meng, E. C. and Ferrin, T. E. (2004) UCSF Chimera--a visualization system for exploratory research and analysis. J. Comput. Chem. 25, 1605-1612. 


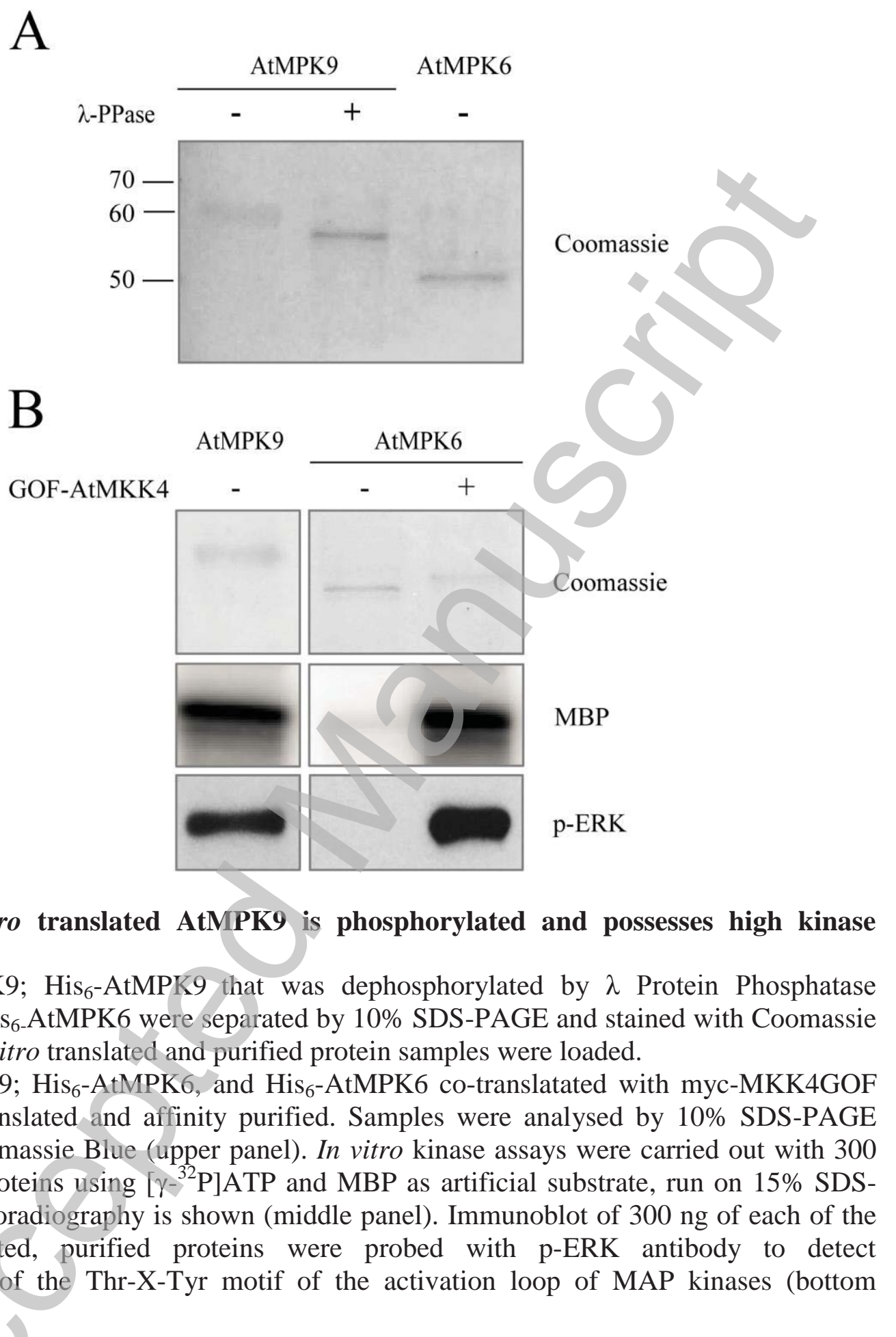

Figure 1 In vitro translated AtMPK9 is phosphorylated and possesses high kinase activity.

(A) His $_{6}$-AtMPK9; His 6 -AtMPK9 that was dephosphorylated by $\lambda$ Protein Phosphatase treatment, and $\mathrm{His}_{6}$-AtMPK6 were separated by 10\% SDS-PAGE and stained with Coomassie Blue. $1 \mu \mathrm{g}$ of in vitro translated and purified protein samples were loaded.

(B) His $_{6}$-AtMPK9; His 6 -AtMPK6, and His 6 -AtMPK6 co-translatated with myc-MKK4GOF were in vitro translated and affinity purified. Samples were analysed by $10 \%$ SDS-PAGE stained with Coomassie Blue (upper panel). In vitro kinase assays were carried out with 300 ng of purified proteins using $\left[\gamma-{ }^{32} \mathrm{P}\right] \mathrm{ATP}$ and MBP as artificial substrate, run on 15\% SDSPAG and the autoradiography is shown (middle panel). Immunoblot of $300 \mathrm{ng}$ of each of the in vitro translated, purified proteins were probed with p-ERK antibody to detect phosphorylation of the Thr-X-Tyr motif of the activation loop of MAP kinases (bottom panel). 
A

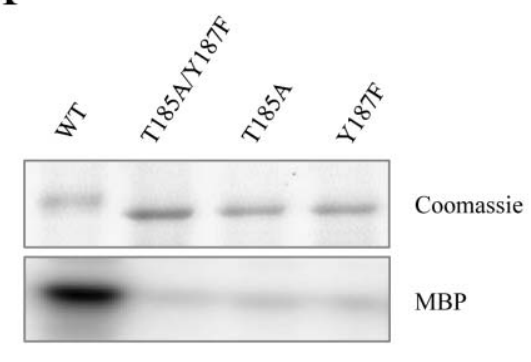

$\mathrm{B}$

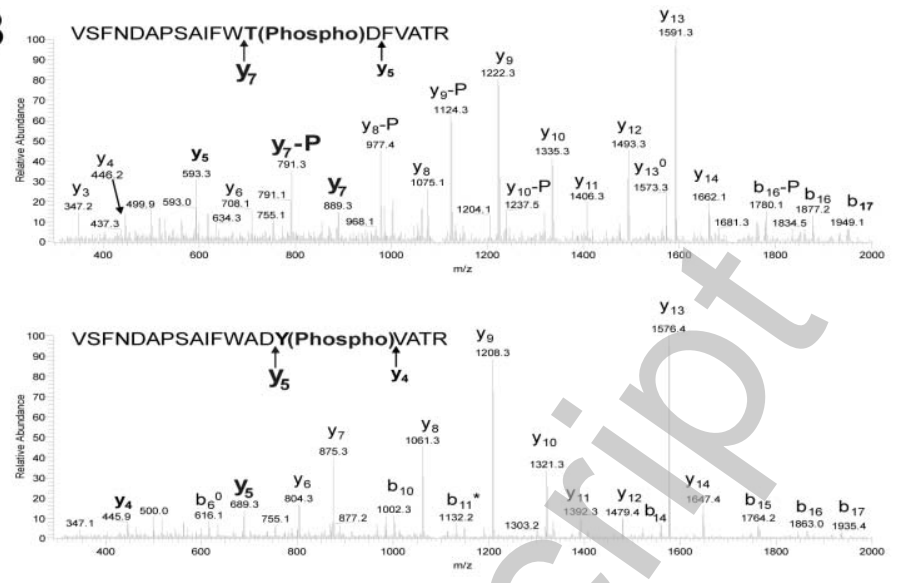

Figure 2 AtMPK9 mutants of the phosphoacceptor sites in the TDY motif of the T-loop are all inactive

(A) $1 \mu \mathrm{g}$ in vitro translated and metal chelate affinity purified His ${ }_{6}-$ AtMPK9 $_{\text {(WT) and T-loop }}$ phosphorylation site mutant forms $\mathrm{His}_{6}$-AtMPK9-T185A/Y187F, His $_{6}$-AtMPK9-T185A, His $_{6}$-AtMPK9-Y187F were separated by $10 \%$ SDS-PAGE and stained with Coomassie Blue (upper panel). $300 \mathrm{ng}$ of in vitro translated and purified proteins were subjected to in vitro kinase assay and analysed as decribed in Figure 1 (lower panel).

(B) TDY triplet corresponding phosphopeptides identified in the in vitro translated and purified AtMPK9 mutants. Collision induced dissociation (CID) spectra of

Upper panel: m/z: $1112.5(2+)$ representing VSFNDAPSAIFWT(Phospho)DFVATR in the Tyr-187 mutant protein. The phosphorylation site is Thr-185 (see fragment ions $\mathrm{y}_{5}$ (unmodified) and $\mathrm{y}_{7}$ (phosphorylated ).

Lower panel: m/z: 1105.5 (2+) representing VSFNDAPSAIFWADY(Phospho)VATR in the Thr-185 mutant protein. Site of phosphorylation is Tyr-187 (see fragment ions $\mathrm{y}_{4}$ (unmodified) and $\mathrm{y}_{5}$ (phosphorylated ). Peptide fragments are labeled according to the nomenclature of Biemann et al [33]. Marks ${ }^{0}$ and $*$ indicate water and ammonia loss from the corresponding fragment ions, respectively. P stands for the 98-Da neutral loss of phosphoric acid characteristic of Ser/Thr phosphorylation. 
AtMPK9

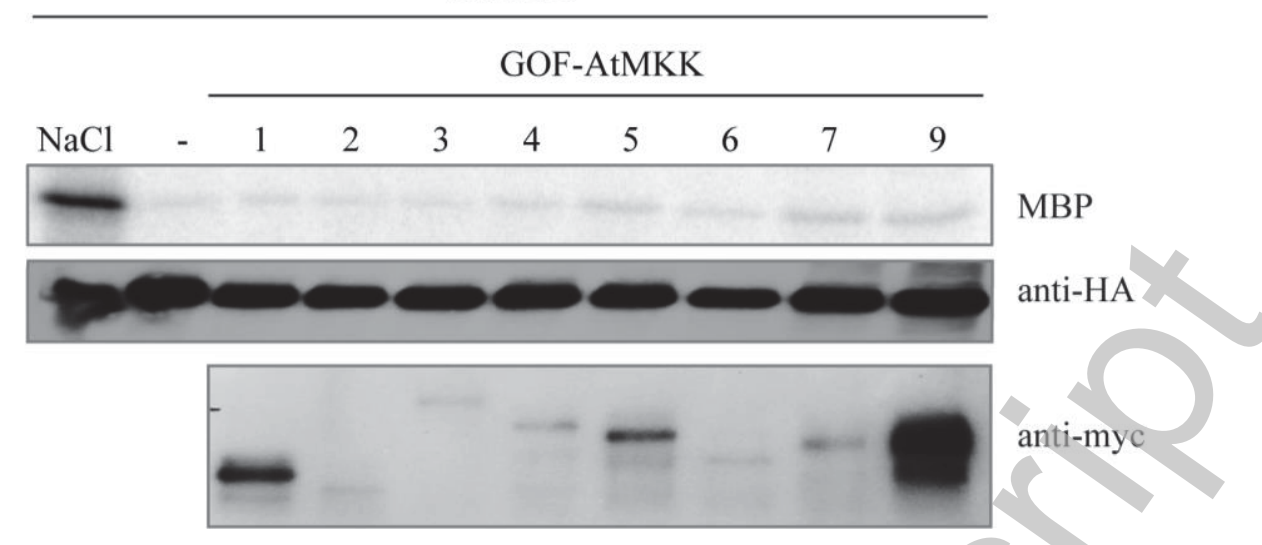

Figure 3 AtMPK9 is activated by salt treatment but not by any of the Arabidopsis gainof-function MKKs in vivo in transformed cultured cells

AtMPK9-HA was transformed into Arabidopsis protoplasts, cells were treated with $250 \mathrm{mM}$ $\mathrm{NaCl}$ for $10 \mathrm{~min}$ (lane 1) or a panel of gain-of-function (GOF). myc-GOF-MKKs (GOFMKK1, GOF-MKK2, GOF-MKK3, GOF-MKK4, GOF-MKK5, GOF-MKK6, GOF-MKK7, GOF-MKK9) were co-transformed with AtMPK9-HA. Transformed cells were harvested after 16 hours incubation. In vitro kinase activity of HA antibody immunoprecipitated AtMPK9 was measured as in Figure 1 (upper panel). $30 \mu \mathrm{g}$ of total protein was analysed by immunoblotting with anti-HA and anti-c-myc antibodies. 


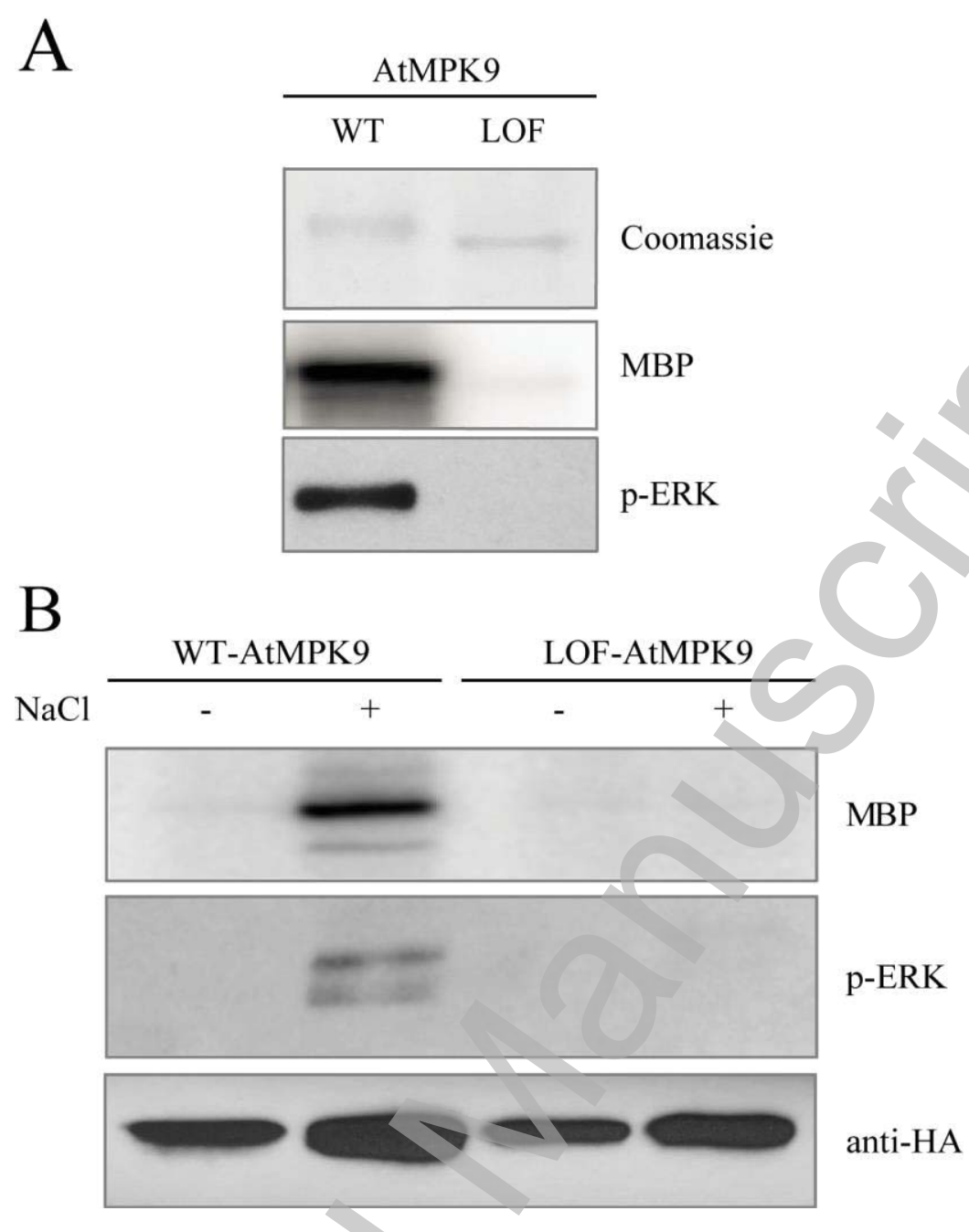

Figure 4 T-loop phosphorylation pattern of in vitro translated and protoplast expressed AtMPK9 suggests autocatalytic activation mechanism

(A) In vitro translated and $1 \mu \mathrm{g}$ purified wild-type His ${ }_{6}$-AtMPK9 (WT) and its kinase inactive mutant form (LOF) His $_{6}$-AtMPK9 were analysed on 10\% SDS-PAG with Coomassie Blue staining (upper panel). Kinase assay was performed with $300 \mathrm{ng}$ affinity purified proteins (middle panel). Phosphorylation of the TDY motif was detected by immunoblotting with the p-ERK antibody (bottom panel).

(B) Wild-type (WT) and loss-of-function (LOF) AtMPK9-HA constructs were transformed into Arabidopsis protoplasts and activated or not by $250 \mathrm{mM} \mathrm{NaCl}$. AtMPK9 was immunoprecipitated with anti-HA-agarose from $30 \mu \mathrm{g}$ of total protein extract and kinase activity was measured (upper panel). Phosphorylation of the TDY motif was detected by immunoblotting with the p-ERK antibody using $30 \mu \mathrm{g}$ of total protein extract (middle panel). AtMPK9-HA was detected by immunoblot using peroxidase conjugated HA antibody (bottom panel). 

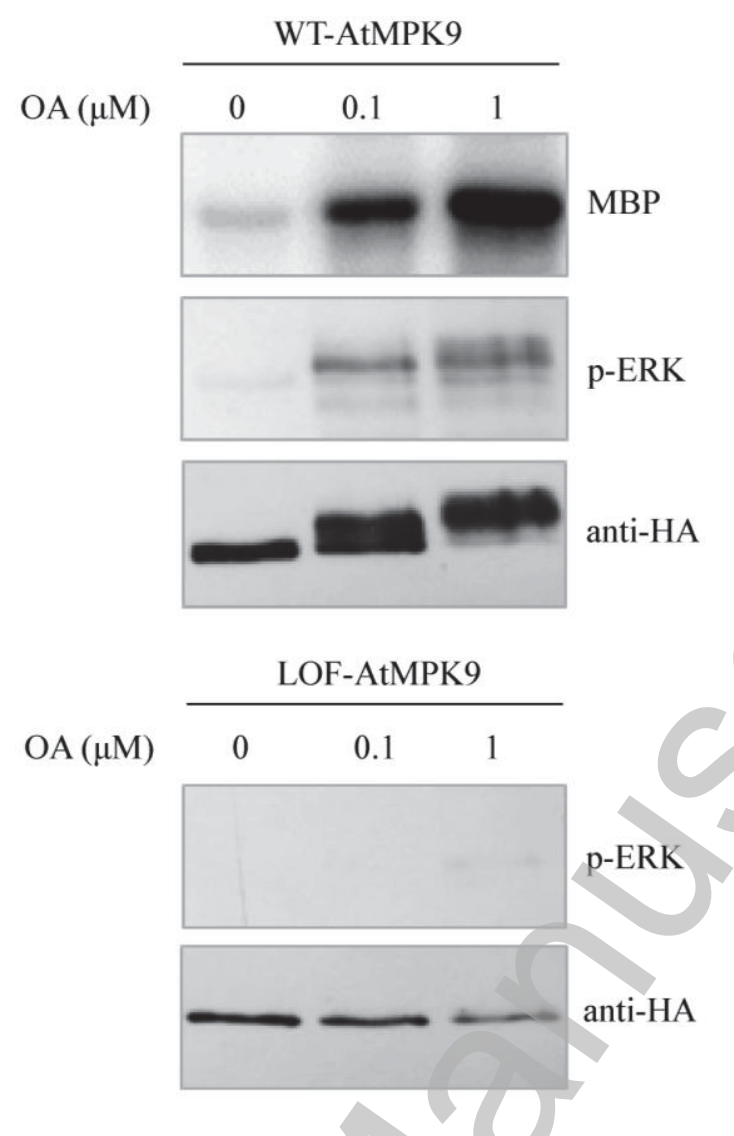

Figure 5 Phosphatase inhibitor, okadaic acid treatment results in phosphorylation and activation of protoplast expressed wild-type AtMPK9 but not the kinase inactive mutant form

Wild-type and kinase inactive mutant forms of AtMPK9 were expressed in protoplasts. Following 1 hour okadaic acid treatment, $30 \mu \mathrm{g}$ of total protein was analysed by immunoblotting with p-ERK and HA antibodies, and in vitro kinase activity of immunoprecipitated AtMPK9 was measured. 


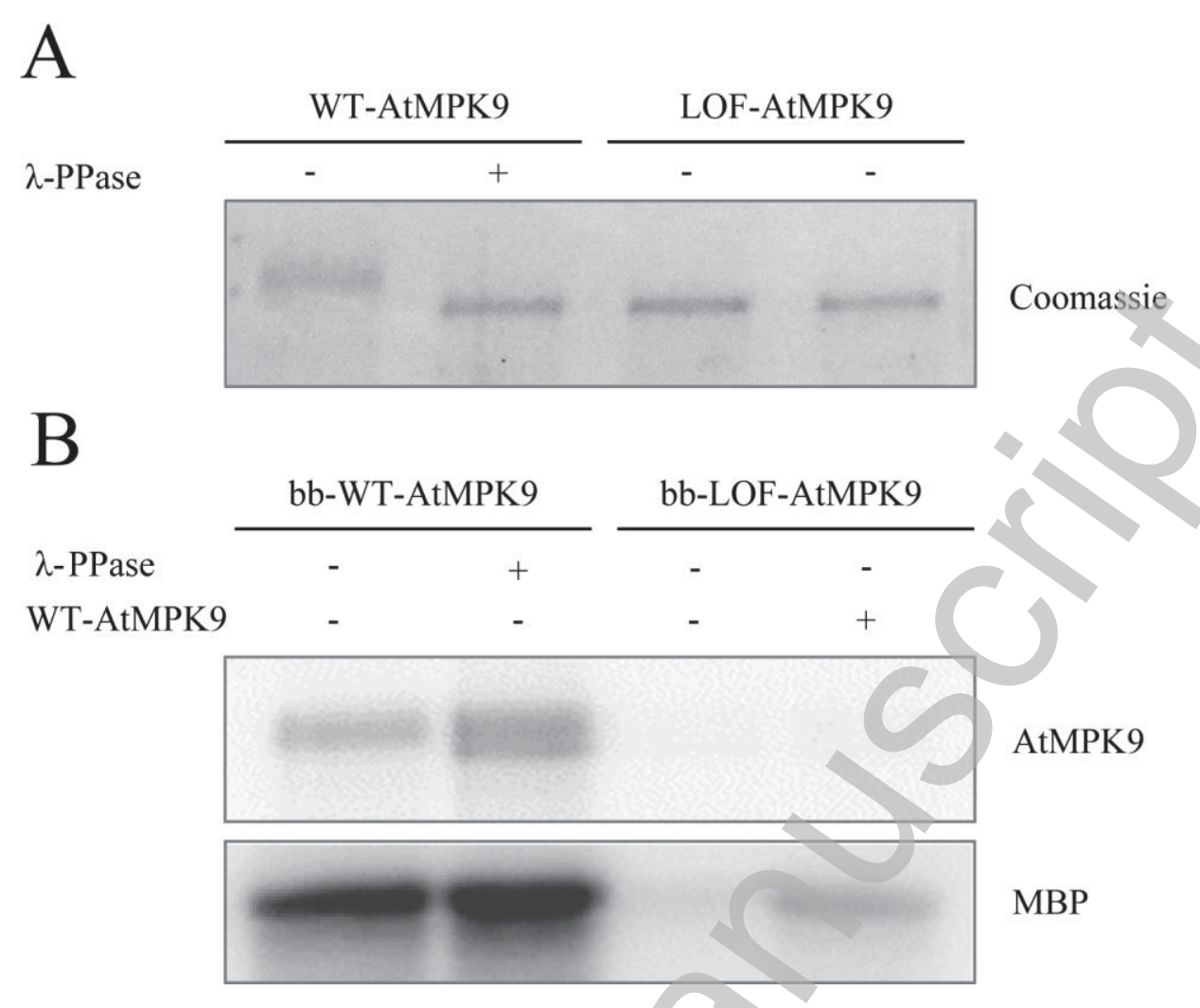

Figure 6 The in vitro translated AtMPK9 is phosphorylated by an intramolecular mechanism

(A) Wild-type (WT) and kinase inactive mutant (LOF) forms of His 6 -AtMPK9 were synthesized by in vitro translation, affinity purified, and treated or not with $\lambda$ Protein Phosphatase. $1 \mu \mathrm{g}$ of proteins were separated by SDS-PAGE and stained with Coomassie Blue.

(B) In vitro kinase assay was performed with 300 ng affinity bead bound wild-type, dephosphorylated wild-type and loss of function AtMPK9 samples to test autophoshorylation. Transphosphorylation was also assessed by the addition of $50 \mathrm{ng}$ of WT-AtMPK9 to $500 \mathrm{ng}$ bead bound inactive $\mathrm{His}_{6}$-LOF-AtMPK9 (last lane, +). Samples were separated on 10\% SDSPAGE and evaluated by autoradiography. In vitro activity of the kinases was tested by using MBP as artificial substrate. 


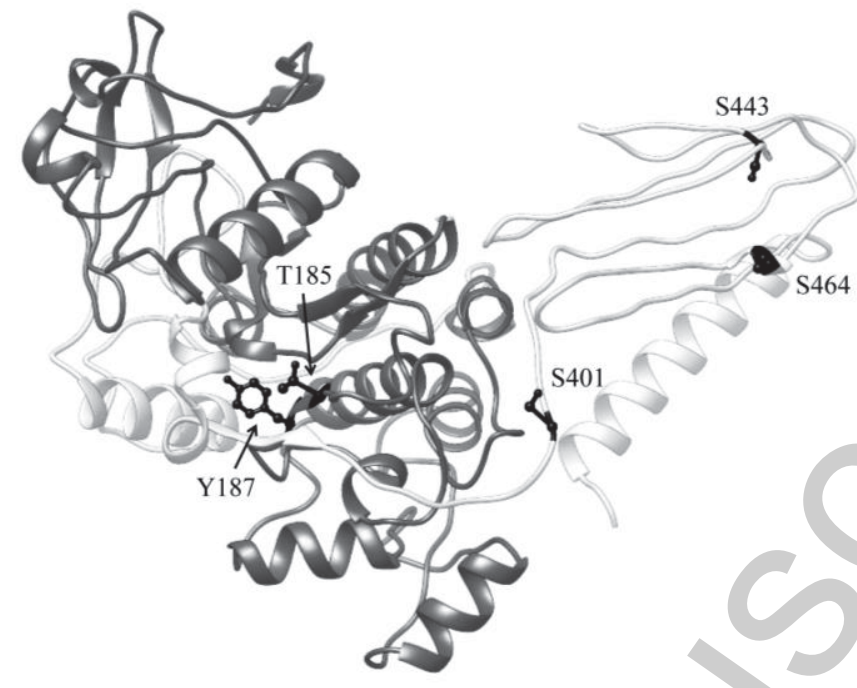

Figure 7 Identified phosphorylated amino acid residues of AtMPK9

The 3D structure of AtMPK9 was generated by I-TASSER online prediction program [34]. Images were rendered using UCSF Chimera [35]. Phosphorylated amino acid residues (Thr185, Tyr187, Ser401, Ser443, Ser464) are indicated with black, kinase domain (1-314 AA) with dark grey and the C-terminal domain with light grey colour. 